\title{
Probing few-particle Laughlin states of photons via correlation measurements
}

\author{
R. O. Umucalılar* and M. Wouters \\ TQC, Universiteit Antwerpen, Universiteitsplein 1, B-2610 Antwerpen, Belgium \\ I. Carusotto \\ INO-CNR BEC Center and Dipartimento di Fisica, Università di Trento, I-38123 Povo, Italy
}

(Received 29 November 2013; published 5 February 2014)

\begin{abstract}
We propose methods to create and observe Laughlin-like states of photons in a strongly nonlinear optical cavity. Such states of strongly interacting photons can be prepared by pumping the cavity with a Laguerre-Gauss beam, which has a well-defined orbital angular momentum per photon. The Laughlin-like states appear as sharp resonances in the particle-number-resolved transmission spectrum. Power spectrum and second-order correlation function measurements yield unambiguous signatures of these few-particle strongly correlated states.
\end{abstract}

DOI: 10.1103/PhysRevA.89.023803

PACS number(s): 42.50.Pq, 42.50.Ar, 42.50.Ct, 73.43.-f

\section{INTRODUCTION}

The goal of creating artificial gauge fields for neutral quantum particles has been a long-sought one for the last two decades of physics research. First attempts inspired by the analogy between the Coriolis force for ultracold atoms in a rotating condensate and the Lorentz force for charged particles in a magnetic field [1,2] culminated in sophisticated methods of imposing a Berry phase on neutral atoms coupling the internal and motional degrees of freedom $[3,4]$.

Recently quantum fluids of light have emerged as a prolific platform to study the condensation phenomena and quantum many-body physics in optical systems [5]. Certain advantages over the cold-atom systems like higher operational temperatures and versatile quantum optical detection techniques make these systems very attractive. Simulating artificial gauge fields for light has also been an active research area for the last couple of years. Among the diverse configurations considered so far, we may count gyromagnetic photonic crystals [6,7], arrays of coupled optical cavities confining single atoms [8,9], microwave circuit-QED devices [10], and solid-state photonic devices operating in the visible or infrared spectral range [1117]. Analogs of the integer quantum Hall edge states were indeed observed in several of these systems $[7,12,17]$.

The prospect of inducing strong interactions between photons opens up the possibility to investigate fractional quantum Hall $(\mathrm{FQH})$ physics in optical systems experiencing an artificial magnetic field [8,9,18-20]. Some promising systems where photons are made to strongly interact with each other via the optical nonlinearity of the underlying medium include a cloud of optically dressed atoms in a Rydberg EIT configuration [21], and in a solid-state context, quantum wells with excitonic optical transitions strongly coupled to the cavity photon [5,22].

In this article, expanding on our previous work [23], we revisit the method of injecting rotating photons into a nonlinear cavity in order to resonantly excite strongly correlated fewparticle states, which are bosonic analogs [24,25] of the usual electronic FQH states, including the Laughlin state $[26,27]$. It is important to note that as opposed to standard quantum

\footnotetext{
*rifatonur.umucalilar@uantwerpen.be
}

optical experiments where the nonlinearity can be accounted for at a perturbative level using a mean-field description, here we propose to look into the eigenstates of the full interacting Hamiltonian. By thoroughly investigating the steady state of the driven-dissipative system for small number of particles, we suggest that unambiguous signatures of strong correlations could be obtained from particle-number-resolved transmission spectra, power spectrum, and second-order correlation function measurements.

\section{SYSTEM HAMILTONIAN AND THE STEADY-STATE DENSITY MATRIX}

The system we consider is a single cavity bounded by spherical mirrors containing a slab of an optically nonlinear medium as sketched in Fig. 1 (see Ref. [23] for details). We assume that the system is cylindrically symmetric around the $z$ axis and the motion is confined to the $x y$ plane of the slab. The Hamiltonian can thus be written using the two-dimensional bosonic field operator $\hat{\Psi}(\mathbf{r})$ as follows:

$$
\begin{aligned}
\mathcal{H}= & \mathcal{H}_{0}+\mathcal{H}_{F} \\
= & \int d^{2} \mathbf{r}\left\{\left[\hat{\Psi}^{\dagger}(\mathbf{r})\left(-\frac{\hbar^{2}}{2 m_{\mathrm{ph}}} \nabla^{2}+\frac{m_{\mathrm{ph}} \omega^{2} r^{2}}{2}+\hbar \omega_{c}\right) \hat{\Psi}(\mathbf{r})\right.\right. \\
& \left.+\frac{\hbar g_{\mathrm{nl}}}{2} \hat{\Psi}^{\dagger}(\mathbf{r}) \hat{\Psi}^{\dagger}(\mathbf{r}) \hat{\Psi}(\mathbf{r}) \hat{\Psi}(\mathbf{r})\right] \\
& \left.+\left[\hbar F(\mathbf{r}, t) \hat{\Psi}^{\dagger}(\mathbf{r})+\hbar F^{*}(\mathbf{r}, t) \hat{\Psi}(\mathbf{r})\right]\right\},
\end{aligned}
$$

where the first square-bracket term is the isolated system Hamiltonian $\mathcal{H}_{0}$ and the second term $\mathcal{H}_{F}$ describes the driving laser field incident on the cavity.

In $\mathcal{H}_{0}$, the finite photon rest frequency $\omega_{c}$ and mass $m_{\mathrm{ph}}=\hbar \omega_{c} / c^{2}$ result from the confinement of photons between the mirrors, while the harmonic trapping with frequency $\omega$ is provided by the mirror curvature [28]. The effective repulsive contact interaction between photons is quantified by $g_{\text {nl }}$ which is proportional to the $\chi^{(3)}$ nonlinearity of the underlying medium [5]. In $\mathcal{H}_{F}$ we take the spatiotemporal profile $F(\mathbf{r}, t)$ of the driving pump to be that of a monochromatic pump with frequency $\omega_{p}$ and normalized amplitude $F$, having the spatial 


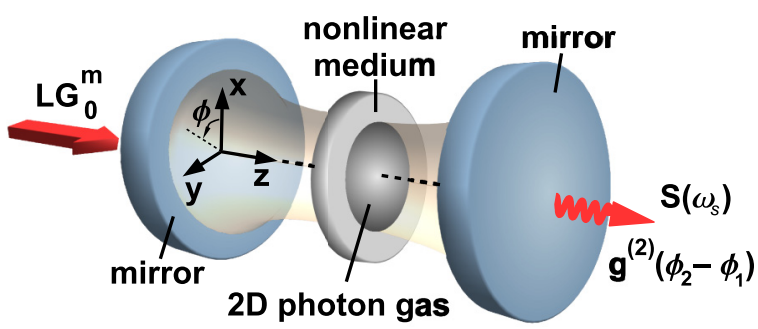

FIG. 1. (Color online) A sketch of the proposed experimental setup. Photons are harmonically trapped inside an optically nonlinear slab with the help of a pair of curved mirrors. A Laguerre-Gauss $\mathrm{LG}_{0}^{m}$ pump beam is used to inject photons each having an orbital angular momentum of $m \hbar$. Photons emitted from the cavity are collected for measurement.

profile of a Laguerre-Gauss beam $\mathrm{LG}_{0}^{m}$ centered on the $z$ axis with orbital angular momentum $m \hbar$.

Finally, radiative and nonradiative photon losses at a rate $\gamma$ can be described through a master equation for the density operator $\hat{\rho}$ in the Lindblad form [29]:

$$
\begin{aligned}
\frac{\partial \hat{\rho}}{\partial t}= & -\frac{i}{\hbar}[\mathcal{H}, \hat{\rho}]+\gamma \int d^{2} \mathbf{r}\left\{\hat{\Psi}(\mathbf{r}) \hat{\rho} \hat{\Psi}^{\dagger}(\mathbf{r})\right. \\
& \left.-\frac{1}{2}\left[\hat{\Psi}^{\dagger}(\mathbf{r}) \hat{\Psi}(\mathbf{r}) \hat{\rho}+\hat{\rho} \hat{\Psi}^{\dagger}(\mathbf{r}) \hat{\Psi}(\mathbf{r})\right]\right\} .
\end{aligned}
$$

The main consequence of losses in the transmission spectra is the broadening of each peak by an amount proportional to $\gamma$ times the number of particles in the corresponding state.

As a first step, a simple understanding of the physical properties of the system can be obtained by moving to a frame rotating around the $z$ axis at angular speed $\Omega$. When seen from this frame, the isolated system Hamiltonian transforms as $\mathcal{H}_{0}^{\text {rot }}=\mathcal{H}_{0}-\int d^{2} \mathbf{r} \hat{\Psi}^{\dagger}(\mathbf{r}) \Omega \mathcal{L}_{z} \hat{\Psi}(\mathbf{r}), \mathcal{L}_{z}=-i \hbar(\mathbf{r} \times \nabla)_{z}$ being the $z$ component of the angular momentum operator [30]. Straightforward manipulation shows that this Hamiltonian can be rewritten as

$$
\begin{aligned}
\mathcal{H}_{0}^{\mathrm{rot}}= & \int d^{2} \mathbf{r}\left\{\hat { \Psi } ^ { \dagger } ( \mathbf { r } ) \left[\frac{(-i \hbar \nabla-\mathbf{A})^{2}}{2 m_{\mathrm{ph}}}+\frac{m_{\mathrm{ph}}\left(\omega^{2}-\Omega^{2}\right) r^{2}}{2}\right.\right. \\
& \left.\left.+\hbar \omega_{c}\right] \hat{\Psi}(\mathbf{r})+\frac{\hbar g_{\mathrm{nl}}}{2} \hat{\Psi}^{\dagger}(\mathbf{r}) \hat{\Psi}^{\dagger}(\mathbf{r}) \hat{\Psi}(\mathbf{r}) \hat{\Psi}(\mathbf{r})\right\}
\end{aligned}
$$

with the vector potential $\mathbf{A}=m_{\mathrm{ph}} \Omega \hat{\mathbf{z}} \times \mathbf{r}$, which leads to a uniform effective magnetic field of magnitude $B=2 m_{\mathrm{ph}} \Omega$ along the $z$ direction for a particle with unit charge. For an angular speed $\Omega$ sufficiently close to the trap frequency $\omega$, it is well known that the ground state of $\mathcal{H}_{0}^{\text {rot }}$ is the bosonic Laughlin $v=1 / 2$ state of the fractional quantum Hall physics $[1,2,24$ 27]:

$$
\Psi_{\mathrm{FQH}}\left(z_{1}, \ldots, z_{N}\right) \propto \prod_{j<k}\left(z_{j}-z_{k}\right)^{2} e^{-\sum_{i=1}^{N}\left|z_{i}\right|^{2} / 2},
$$

where $z_{j}=\left(x_{j}+i y_{j}\right) / \ell$ is the complex coordinate of the $j$ th particle in units of the oscillator length $\ell=\sqrt{\hbar / m_{\mathrm{ph}} \omega}$. This $N$-particle wave function is composed of single-particle wave functions in the lowest Landau level (LLL) and is an eigenfunction of the total angular momentum operator $\hat{L}_{z}$ with eigenvalue $L_{z}=N(N-1) \hbar$.
While working in the rotating frame has been useful to establish the analogy between $\mathcal{H}_{0}^{\text {rot }}$ and the fractional quantum Hall Hamiltonian for charged particles in a real magnetic field, all optical observations are actually performed in the laboratory frame. In this frame, the Laughlin state represented by the wave function (4) keeps being the nondegenerate ground state of the isolated system Hamiltonian $\mathcal{H}_{0}$ for a given angular momentum and is separated from excited states of the same total angular momentum by an excitation gap of the order of the lowest Haldane pseudopotential $v_{0}=\hbar g_{\mathrm{nl}} / 2 \pi \ell^{2}$ for the contact potential, which is basically the interaction energy of two particles in the LLL with zero relative angular momentum (cf. Appendix A and Refs. [27,31]). In order to prevent Landau-level mixing and restrict the description to the LLL, we will require $v_{0} \ll \hbar \omega$. Since the particles in the Laughlin state do not feel any interaction, the total energy in the rotating frame is simply the energy of $N$ noninteracting particles in the LLL shifted by the cavity rest frequency $\hbar \omega_{c}$ : These two contributions sum up to give a total energy of $N \hbar\left(\omega+\omega_{c}\right)$. After moving to the laboratory frame, the energy becomes $E_{N ; L_{z}}=N \hbar\left(\omega+\omega_{c}\right)+\omega L_{z}=N^{2} \hbar \omega+N \hbar \omega_{c}$.

In order to efficiently prepare a Laughlin state of photons in the desired $N$-particle sector, one therefore has to set the pump frequency $\omega_{p}$ to $E_{N ; L_{z}} / N \hbar=N \omega+\omega_{c}$ and the angular momentum per photon $m \hbar$ of the Laguerre-Gauss mode $\mathrm{LG}_{0}^{m}$ to $L_{z} / N=(N-1) \hbar$ [23]. Provided the excitation gap $v_{0}$ is larger than the linewidth $N \gamma$ of the state, the optical pump will be able to selectively excite the Laughlin state.

Due to the coherent nature of the laser drive, the steady state of the system will be a superposition of states with different number of particles, which in the weak driving limit $\tilde{F} \equiv$ $\ell F / \gamma \ll 1$ can formally be written as $|\Psi\rangle=\sum_{N=0}^{\infty} c_{N} \tilde{F}^{N}|N\rangle$, where $|N\rangle$ denotes an $N$-particle state and $c_{N}$ are constants of $O(1)$ [20]. Thus the probability $P_{N}$ of having an $N$-particle state in the system scales as $\tilde{F}^{2 N}$. This probability can be measured by detecting $N$ transmitted photons simultaneously, which rules out the possibility that the detected state has a smaller number of particles $N^{\prime}<N$. The contribution of states with a larger number of particles $N^{\prime}>N$ is already suppressed by a factor of $\tilde{F}^{2\left(N^{\prime}-N\right)}$ due to the weak driving condition $\tilde{F} \ll 1$. In Figs. 2(a) and 3(a) we show the ratio $P_{N} / P_{N-1}$ as a function of pump detuning $\Delta \omega_{p}=\omega_{p}-\omega_{c}$ for $N=2,3$ in the presence of Laguerre-Gauss $\mathrm{LG}_{0}^{1}, \mathrm{LG}_{0}^{2}$ driving modes, respectively. The probability $P_{N}=\operatorname{Tr}\left(\Pi_{N} \rho_{\mathrm{ss}}\right), \Pi_{N}$ being the projector onto the $N$-particle subspace, is calculated by using the steady-state density matrix $\rho_{\mathrm{ss}}$ found via a superoperator approach to solve the master equation (2) [19]. Plotting the ratio $P_{N} / P_{N-1}$ eliminates the effect of intermediate states with a smaller number of particles $N^{\prime}<N$ on the transmission spectrum, leading to clear resonance peaks corresponding to $N$-particle eigenstates of the system.

In order to see how faithfully the eigenstates are reproduced we plotted in Figs. 2(b) and 3(b) the overlap $\mathcal{O}\left(\Psi^{(N)}, \Phi\right)=$ $\left|\left\langle\Psi^{(N)} \mid \Phi\right\rangle\right|^{2} /\left\langle\Psi^{(N)} \mid \Psi^{(N)}\right\rangle\langle\Phi \mid \Phi\rangle$ between the $N$-photon amplitude $\Phi\left(z_{1}, \ldots, z_{N}\right)=\operatorname{Tr}\left[\hat{\Psi}\left(z_{1}\right) \ldots \hat{\Psi}\left(z_{N}\right) \rho_{s s}\right]$ and the $N$-particle eigenstates $\Psi^{(N)}$ for the well-resolved peaks corresponding to the lowest- and highest-energy eigenstates. While the lowest-energy eigenfunction for total angular momentum $N(N-1) \hbar$ is the Laughlin wave function (4), the 

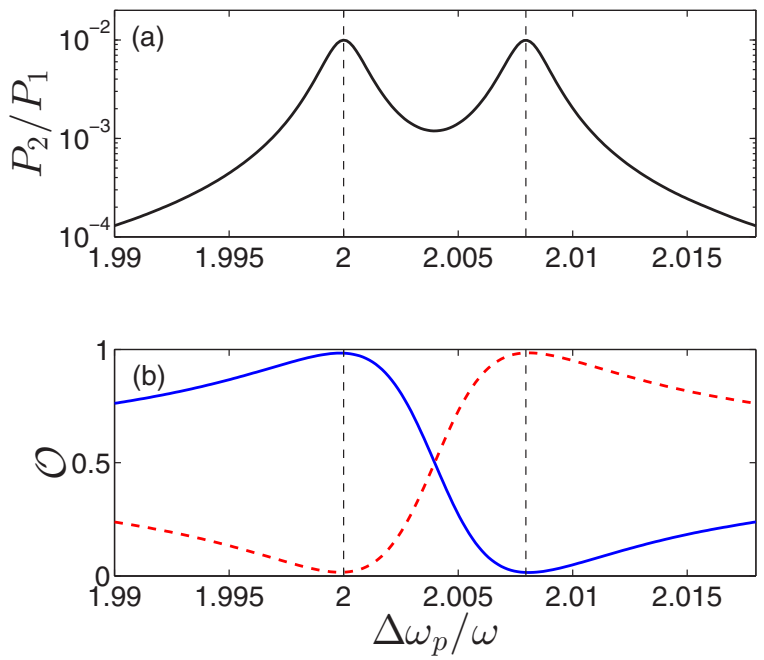

FIG. 2. (Color online) (a) Ratio $P_{2} / P_{1}$ of having two particles to one particle in the steady state as a function of the detuning of pump frequency $\Delta \omega_{p}$ in the presence of a Laguerre-Gauss $\mathrm{LG}_{0}^{1}$ pump. (b) The overlap $\mathcal{O}$ between the two-photon amplitude and two-particle eigenfunctions as a function of $\Delta \omega_{p}$ (solid blue line for the Laughlin state, dashed red line for the c.m. state). Vertical dashed lines correspond to half the two-particle eigenfrequencies of the isolated system Hamiltonian $\mathcal{H}_{0}$. System and pump parameters: $g_{\mathrm{nl}} / \ell^{2} \omega=0.1, \gamma / \omega=0.002$, and $\ell F / \gamma=0.1$.

highest-energy eigenfunction is found to be

$$
\Psi_{\text {c.m. }}\left(z_{1}, \ldots, z_{N}\right) \propto\left(\sum_{i=1}^{N} z_{i}\right)^{N(N-1)} e^{-\sum_{i=1}^{N}\left|z_{i}\right|^{2} / 2},
$$
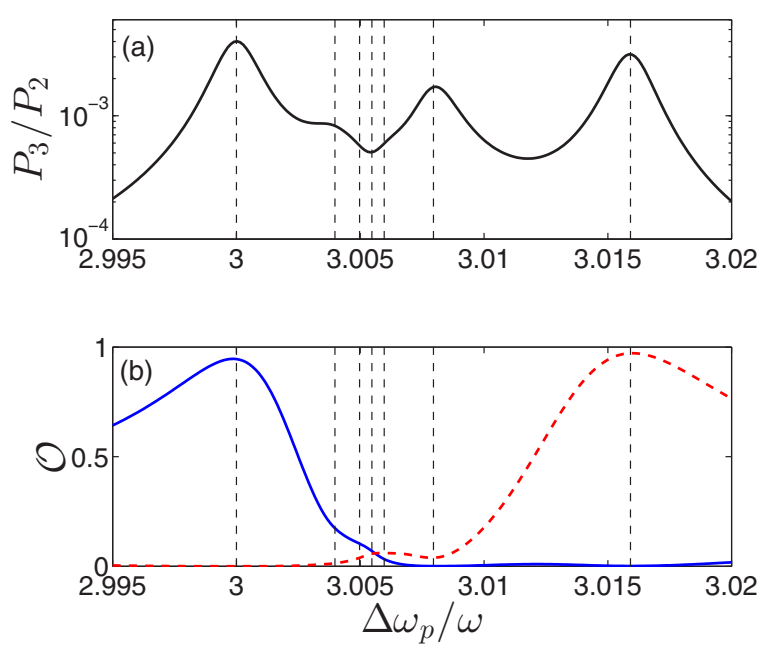

FIG. 3. (Color online) (a) Ratio $P_{3} / P_{2}$ of having three particles to two particles in the steady state as a function of the detuning of pump frequency $\Delta \omega_{p}$ in the presence of a Laguerre-Gauss $L_{0}^{2}$ pump. (b) The overlap $\mathcal{O}$ between the three-photon amplitude and three-particle eigenfunctions as a function of $\Delta \omega_{p}$ (solid blue line for the Laughlin state, dashed red line for the c.m. state). Vertical dashed lines correspond to one third of the three-particle eigenfrequencies of the isolated system Hamiltonian $\mathcal{H}_{0}$. System and pump parameters: $g_{\mathrm{nl}} / \ell^{2} \omega=0.1, \gamma / \omega=0.002$, and $\ell F / \gamma=0.1$. corresponding to pure center-of-mass (c.m.) rotation (cf. Appendix A). The overlaps larger than $95 \%$ on resonance confirm that the present excitation scheme is indeed successful in generating the target eigenstates with very good fidelity. An obvious way to further improve the fidelity is to decrease the loss rate $\gamma$, which would help to prevent spurious excitation of nearby states. For the specific case of the Laughlin state the condition to avoid this can be quantified roughly as $\gamma \ll$ $g_{\mathrm{nl}} / 2 \pi N \ell^{2}$, meaning that the dissipation induced broadening should be sufficiently smaller than the interaction induced excitation gap. Note that this condition is only marginally satisfied for the parameters in the figures, still the fidelity is quite close to 1 .

To better understand the system at hand and investigate its properties further it will prove to be useful to examine the steady-state density matrix $\rho_{s s}$ from a semianalytical perspective. To facilitate the notation we will use the occupation number representation $\left|n_{0} n_{1} \cdots n_{m} \cdots\right\rangle$, where $n_{m}$ is the number of particles in the single-particle LLL state with angular momentum $m \hbar$ and wave function $\varphi_{m}(z)=$ $z^{m} e^{-|z|^{2} / 2} / \sqrt{\pi m !}$. Focusing on the case where the system is pumped by a Laguerre-Gauss $L^{1}$ beam we make the following ansatz for the steady state of the system, keeping states with $N=0,1,2$ particles:

$$
\begin{aligned}
|\Psi\rangle \simeq & c_{0}|000\rangle+c_{1} \tilde{F}|010\rangle \\
& +c_{2} \tilde{F}^{2}[a(|101\rangle-|020\rangle)+b(|101\rangle+|020\rangle)],
\end{aligned}
$$

where $c_{0}, c_{1}$, and $c_{2}$ are complex constants with magnitude of $O(1)$. Since $\tilde{F} \ll 1,|\Psi\rangle$ will mainly be the vacuum state $|000\rangle$. The single-particle state $|010\rangle$ is simply the one associated with a single pump photon with one unit of angular momentum. The two-particle LLL manifold is spanned by the Laughlin state $(|101\rangle-|020\rangle) \leftrightarrow\left(z_{1}-z_{2}\right)^{2}$ and the c.m. state $(|101\rangle+|020\rangle) \leftrightarrow\left(z_{1}+z_{2}\right)^{2}$, with weights $a$ and $b=\sqrt{1-|a|^{2}}(|a| \leqslant 1)$, respectively. In this limit one can approximately construct the corresponding density matrix by forming $|\Psi\rangle\langle\Psi|$. However, we make a better approximation by considering an additional quantum jump term (see, e.g., Ref. [20]) as follows:

$$
\rho_{\mathrm{ss}} \simeq\left(|\Psi\rangle\left\langle\Psi\left|+\mathcal{N} \sum_{m=0}^{2} a_{m}\right| \Psi\right\rangle\langle\Psi| a_{m}^{\dagger}\right),
$$

where $\mathcal{N}$ is a constant of $O(1)$ and $a_{m}$ is the destruction operator for the LLL single-particle state with angular momentum $m \hbar$. To verify the appropriateness of this description, we numerically solved for the steady-state density matrix and compared it with the prediction of (7) after optimizing the variables. For resonant excitation of the Laughlin state at $\Delta \omega_{p} / \omega=2$, with parameters $g_{\mathrm{nl}} / \ell^{2} \omega=0.1, \gamma / \omega=0.002$, and $\tilde{F}=0.1$, we obtained $a \approx-(0.127+0.984 i), b \approx 0.128$, $c_{0} \approx 0.962, c_{1} \approx-(0.005+1.923 i), c_{2} \approx-0.166+1.337 i$, and $\mathcal{N} \simeq 0.999$, yielding a very small weighted absolute percentage error of $\sum_{i j}\left|\rho_{i j}^{\text {num }}-\rho_{i j}^{\text {pre }}\right| / \sum_{i j}\left|\rho_{i j}^{\text {num }}\right| \approx 0.3 \%$ between the numerical and predicted density matrices. Similar results were found for the resonant excitation of the c.m. state at $\Delta \omega_{p} / \omega \approx 2.008$, with the roles of $a$ and $b$ interchanged. Although the contribution of the additional quantum jump term to the density matrix itself is small (error with $\mathcal{N}=0$ 
is $\approx 0.8 \%$ ), its effect on certain observables can be sizable as we shall see in the next section. We finally note that if one is interested in finding the contribution of states with higher number of particles to such observables, it is essential to include quantum jump terms involving the annihilation of more than a single particle.

\section{POWER SPECTRUM}

Although the $N$-photon amplitude could be measured through a combination of several homodyne detections for a direct comparison with a known wave function (see, e.g., Ref. [19]), it is desirable to find a technically simpler observable that would reveal at least some property peculiar to these correlated states. With this aim in mind, we propose to look at the power spectrum $S\left(\mathbf{r}, \omega_{s}\right) \propto \operatorname{Re}\left[\int_{0}^{\infty} g^{(1)}(\mathbf{r}, \tau ; \mathbf{r}, 0) e^{-i \omega_{s} \tau} d \tau\right]$, where $g^{(1)}(\mathbf{r}, \tau ; \mathbf{r}, 0) \equiv \operatorname{Tr}\left[\hat{\Psi}^{\dagger}(\mathbf{r}, \tau) \hat{\Psi}(\mathbf{r}, 0) \rho_{\mathrm{ss}}\right]$ is the first-order correlation function [32]. We calculated $g^{(1)}$ as a function of time delay $\tau$ by numerically evolving the master equation (2) [29]. Figure 4 shows the power spectrum (a) for the resonant excitation of a two-particle Laughlin state and (c) for the resonant excitation of a three-particle Laughlin state. The most prominent feature seen in Figs. 4(a) and 4(c) is the appearance of sharp peaks at integer multiples of the trap frequency $\omega$.
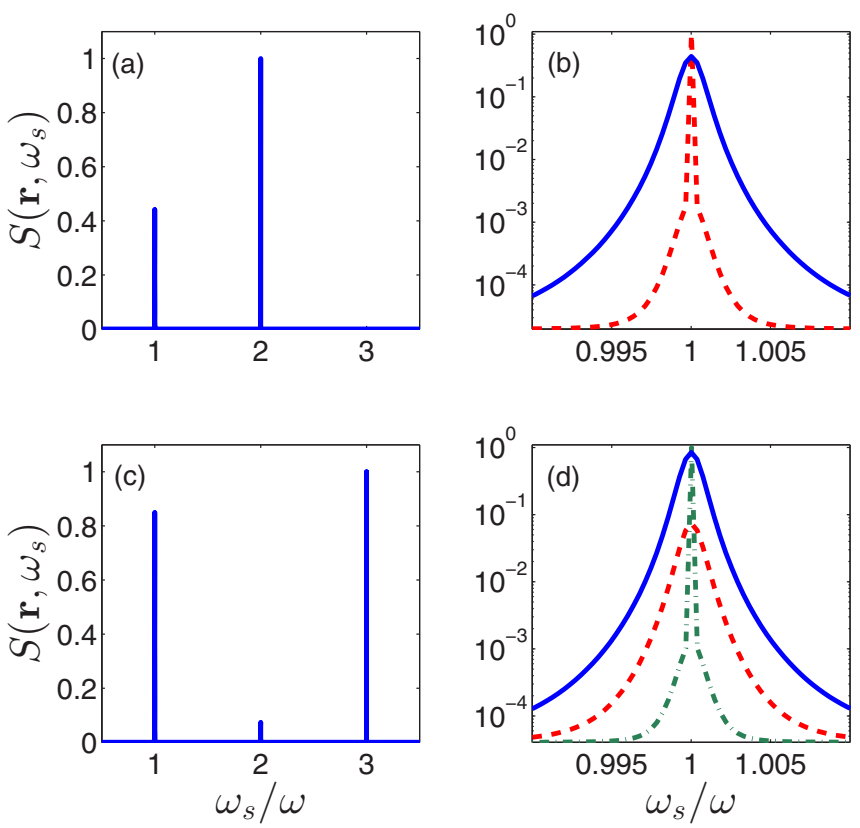

FIG. 4. (Color online) Power spectrum $S\left(\mathbf{r}, \omega_{s}\right)$ in arbitrary units as a function of frequency $\omega_{s}$ measured with respect to the cavity frequency $\omega_{c}$. (a) and (b) The system is pumped by a Laguerre-Gauss $\mathrm{LG}_{0}^{1}$ beam at $\Delta \omega_{p} / \omega=2 ;|\mathbf{r}|=0.05 \ell$. (b) A closeup of the original spectrum (solid blue line) together with the spectrum shifted by -1 (dashed red line) along the frequency axis. (c) and (d) The system is pumped by a Laguerre-Gauss $\mathrm{LG}_{0}^{2}$ beam at $\Delta \omega_{p} / \omega=3 ;|\mathbf{r}|=0.15 \ell$. (d) A closeup of the original spectrum (solid blue line) together with the ones shifted by -1 (dashed red line) and -2 (dash-dotted green line) along the frequency axis. System and pump parameters: $g_{\mathrm{nl}} / \ell^{2} \omega=0.1, \gamma / \omega=0.002$, and $\ell F / \gamma=0.1$.
In order to understand the origin of these peaks it is useful to examine the limit of vanishingly small losses and driving so that the time dependence of an operator can be approximated as $\hat{O}(\tau)=e^{i \mathcal{H}_{0} \tau / \hbar} \hat{O} e^{-i \mathcal{H}_{0} \tau / \hbar}$. Let us for simplicity consider the case of Fig. 4(a), where the system is pumped by an $\mathrm{LG}_{0}^{1}$ beam at $\Delta \omega_{p} / \omega=2$ to be on resonance with a two-particle Laughlin state. Expanding the field operators in the LLL basis we obtain

$$
g^{(1)}(\mathbf{r}, \tau ; \mathbf{r}, 0)=\sum_{i, j} \varphi_{i}^{*}(\mathbf{r}) \varphi_{j}(\mathbf{r}) \operatorname{Tr}\left[a_{i}^{\dagger}(\tau) a_{j} \rho_{\mathrm{ss}}\right] .
$$

Recalling that $E_{N ; L_{z}}-E_{N-1 ; L_{z}-m}=(m+1) \hbar \omega$ and using Eqs. (6) and (7), the individual trace terms are computed as

$$
\begin{gathered}
\operatorname{Tr}\left[a_{0}^{\dagger}(\tau) a_{0} \rho_{\mathrm{ss}}\right]=e^{i \omega \tau}(1+\mathcal{N})\left|c_{2} \tilde{F}^{2}(a+b)\right|^{2} \\
\operatorname{Tr}\left[a_{1}^{\dagger}(\tau) a_{1} \rho_{\mathrm{ss}}\right]=e^{i 2 \omega \tau}\left[\left|c_{1} \tilde{F}\right|^{2}+2(1+\mathcal{N})\left|c_{2} \tilde{F}^{2}(a-b)\right|^{2}\right] \\
\operatorname{Tr}\left[a_{2}^{\dagger}(\tau) a_{2} \rho_{\mathrm{ss}}\right]=e^{i 3 \omega \tau}(1+\mathcal{N})\left|c_{2} \tilde{F}^{2}(a+b)\right|^{2}
\end{gathered}
$$

All off-diagonal terms $\operatorname{Tr}\left[a_{i}^{\dagger}(\tau) a_{j} \rho_{\mathrm{ss}}\right]$ with $i \neq j$ vanish identically. This follows from the fact that a definite particlenumber sector of the state (6) has a well-defined total angular momentum and $a_{i}^{\dagger} a_{j}$ is a particle-number conserving operator associated with a change in total angular momentum by $(i-j) \hbar$. This reasoning also applies to any state $a_{m}|\Psi\rangle$ contributing to the quantum jump term in Eq. (7). Note that each diagonal term $\operatorname{Tr}\left[a_{l}^{\dagger}(\tau) a_{l} \rho_{\mathrm{ss}}\right]$ corresponds to the $g^{(1)}(\tau)$ coherence function of light emitted in a state with angular momentum $l \hbar$, which can be measured by making use of holograms to isolate different angular momentum components.

Terms in Eqs. (9) and (11) appear solely because of interactions, which can be seen by taking $a=-b=-1 / 2$ leading to vanishing traces and to the emergence of the state $|020\rangle$ due to the pump as the only two-particle state in Eq. (6). In the absence of interactions, we numerically confirmed that the only surviving trace is $\operatorname{Tr}\left[a_{1}^{\dagger}(\tau) a_{1} \rho_{\mathrm{ss}}\right]$ corresponding to the pump mode, which leads to a single peak at $\omega_{s} / \omega=2$ in the spectrum. Note also that had we not included the quantum jump term in Eq. (7), i.e., if $\mathcal{N}=0$, the amplitudes in Eqs. (9) and (11) would have been reduced almost twice, given that $\mathcal{N} \sim 1$. In Fig. 4(a) two peaks are seen at $\omega_{s} / \omega=1,2$ corresponding to states with angular momentum $0 \hbar, 1 \hbar$, respectively. The third peak that is actually present at $\omega_{s} / \omega=$ 3 is not visible due to the chosen spatial point $|\mathbf{r}|=0.05 \ell$ as it is suppressed with respect to the peak at $\omega_{s} / \omega=1$ by a factor of the order of $\left|\varphi_{2}(\mathbf{r})\right|^{2} /\left|\varphi_{0}(\mathbf{r})\right|^{2}=|\mathbf{r} / \ell|^{4} / 2$. In Fig. 4(b) the spectrum shifted by -1 along the frequency axis (dashed red line) is superposed onto the original spectrum (solid blue line) for better comparison of the line shapes of the peaks. It is seen that while the interaction-induced peak at $\omega_{s} / \omega=1$ (solid blue line) is broadened by an amount determined by the loss rate $\gamma / \omega=0.002$, the peak at $\omega_{s} / \omega=2$ displays a narrow, $\delta$-like feature on top of a pedestal of width $\gamma$. This narrow feature has a width determined by the finite time window used in the numerical calculation and originates from elastic scattering of the pump.

In Fig. 4(c) we display the power spectrum obtained for an $\mathrm{LG}_{0}^{2}$ pump on resonance with a three-particle Laughlin state at $\Delta \omega_{p} / \omega=3$. While the peak at $\omega_{s} / \omega=3$ appears 
because of the pump, as checked numerically, those at $\omega=1,2$ are due to interactions which scatter particles to different angular momentum states. Figure 4(d) displays the shifted spectra, where it is again possible to observe the radiative broadening of the interaction-induced peaks as opposed to the $\delta$-like elastic pump scattering peak. We numerically verified that in the present weak-excitation limit, quantum jump terms in the steady-state density matrix are indeed negligible and the two-particle sector is well approximated by a single, noninteracting wave function of the form $\Psi\left(z_{1}, z_{2}\right) \propto\left(z_{1}-\right.$ $\left.z_{2}\right)^{2}\left(z_{1}^{2}+z_{2}^{2}+c z_{1} z_{2}\right)$, where the exact value of the parameter $c$ is determined by system parameters.

This observation helps to explain the appearance of peaks only at integer multiples of $\omega$. It suggests that once a singleparticle state in the LLL is resonantly excited, all higher $N$ particle states accessible through driving and losses lie in the lowest energy manifold of the corresponding $N$-particle state as long as the LLL approximation is valid and the $v_{0} \gg \gamma$ condition on the Laughlin gap is satisfied. That is, their wave function can be written as the Laughlin wave function times a symmetric polynomial compatible with the given total angular momentum (cf. Appendix B). This result is to be contrasted to the case of the resonant excitation of the two-particle c.m. state: As it is shown in Appendix $\mathrm{C}$, the power spectrum now displays peaks also at frequencies other than integer multiples of $\omega$.

\section{SECOND-ORDER CORRELATION FUNCTION}

In this section we show that an equal-time second-order correlation function $g^{(2)}$ measurement yields clear signatures of strong correlations which distinguish the Laughlin state from the excited states with same total angular momentum by revealing information about the spatial structure of wave functions.

As a first point, it is crucial to keep in mind that such a measurement is based on the simultaneous detection of two photons. In practice, this means that the time resolution of the detectors has to be high enough that the spatial correlations will not be washed out due to the fast rotation of the Laughlin fluid of light in the trap at frequency $\omega$, giving rise to a rapidly varying $g^{(2)}\left(\mathbf{r}_{1}, t ; \mathbf{r}_{2}, t+\tau\right)$ as a function of time delay $\tau$ on a time scale $\omega^{-1}$.

Should the required temporal resolution be too stringent, one has to find a scheme to compensate the effect of rotation, which in principle should enable one to measure the slowly varying quantity $g^{(2)}\left(\mathbf{r}_{1}, t ; \mathcal{R}_{-\omega \tau}\left[\mathbf{r}_{2}\right], t+\tau\right)$, where $\mathcal{R}_{-\omega \tau}$ is the rotation operator which rotates the coordinate $\mathbf{r}_{2}$ by $-\omega \tau$, undoing the inherent rotation of the system. Recalling the angular momentum as the rotation generator, we see that such a global rotation in time is possible if one can decompose the output field into different angular momentum components and then shift the frequency of each component properly, by using modulators, e.g., acousto-optic modulators (AOMs). This decomposition is also relevant and useful in the present context as each single particle state in the LLL has a definite angular momentum. As a first step, one has to separate these components: To this purpose, there exist angular momentum sorting protocols that have been experimentally demonstrated at the level of a single photon [33]. Alternatively, separation can be performed spectroscopically by using, for instance, a diffraction grating. Each angular momentum channel with angular momentum $l \hbar$ is then let through an AOM which shifts the frequency of the incoming light by $\Delta \omega=-l \omega$. Superimposing again the different components, one recovers the initial field profile after compensating for the unwanted rotation and the $g^{(2)}$ measurement can be performed on a slow detector. Of course, all this manipulation has to be performed in a fully phase-coherent way without spurious distortions of the phase fronts that may disturb interference.

We consider the usual form [32] of the normalized equaltime second-order correlation function

$$
g^{(2)}\left(\mathbf{r}_{1}, \mathbf{r}_{2}\right)=\frac{\operatorname{Tr}\left[\hat{\Psi}^{\dagger}\left(\mathbf{r}_{1}\right) \hat{\Psi}^{\dagger}\left(\mathbf{r}_{2}\right) \hat{\Psi}\left(\mathbf{r}_{2}\right) \hat{\Psi}\left(\mathbf{r}_{1}\right) \rho_{\mathrm{ss}}\right]}{\operatorname{Tr}\left[\hat{\Psi}^{\dagger}\left(\mathbf{r}_{1}\right) \hat{\Psi}\left(\mathbf{r}_{1}\right) \rho_{\mathrm{ss}}\right] \operatorname{Tr}\left[\hat{\Psi}^{\dagger}\left(\mathbf{r}_{2}\right) \hat{\Psi}\left(\mathbf{r}_{2}\right) \rho_{\mathrm{ss}}\right]} .
$$

As done in the previous section for $g^{(1)}$, we can derive an analytical form for $g^{(2)}$ using Eqs. (6) and (7). For coordinates $\mathbf{r}_{1}=\left(r_{\circ}, \phi_{1}\right)$ and $\mathbf{r}_{2}=\left(r_{\circ}, \phi_{2}\right)$ we find

$$
\begin{aligned}
g^{(2)}\left(r_{\circ}, \phi\right)= & \alpha\left(r_{\circ}\right)\left[(1+\cos 2 \phi)|a+b|^{2}\right. \\
& \left.+4 \cos \phi\left(b^{2}-|a|^{2}\right)+2|a-b|^{2}\right],
\end{aligned}
$$

where $\phi=\phi_{2}-\phi_{1}$ and $\alpha\left(r_{\circ}\right)$ is a prefactor depending on the fixed radial coordinate $r_{\circ}$.

In Fig. 5 the numerically evaluated $g^{(2)}(\phi)$ is shown for three different driving frequencies of the $\mathrm{LG}_{0}^{1}$ beam. The solid line in Fig. 5(a) is obtained for $\Delta \omega_{p} / \omega=2$ when the two-particle Laughlin state is resonantly driven. There is strong antibunching at $\phi=0$ as expected, since two particles cannot be in close vicinity of each other in this state. As $\phi \rightarrow \pi$ the degree of anticorrelation is strongly suppressed. Figure 5(b) shows the case for the resonant excitation of the c.m. state at $\Delta \omega_{p} / \omega \approx 2.008$, where a strong bunching effect is observed at $\phi=0$. Again as $\phi \rightarrow \pi$ this correlation effect gradually becomes less pronounced. This marked difference of $g^{(2)}(\phi)$ in two cases is directly related to the fact that the Laughlin wave function $\left(z_{1}-z_{2}\right)^{2}$ becomes the c.m. wave function $\left(z_{1}+z_{2}\right)^{2}$ upon changing $\phi$ to $\phi+\pi$. The huge difference between the maximum amplitudes of $g^{(2)}(\phi)$ on the other hand is due to the prefactor $\alpha\left(r_{\circ}\right)$ in Eq. (13), which is roughly $\left|c_{2}\right|^{2} /\left|c_{1}\right|^{4}$ for
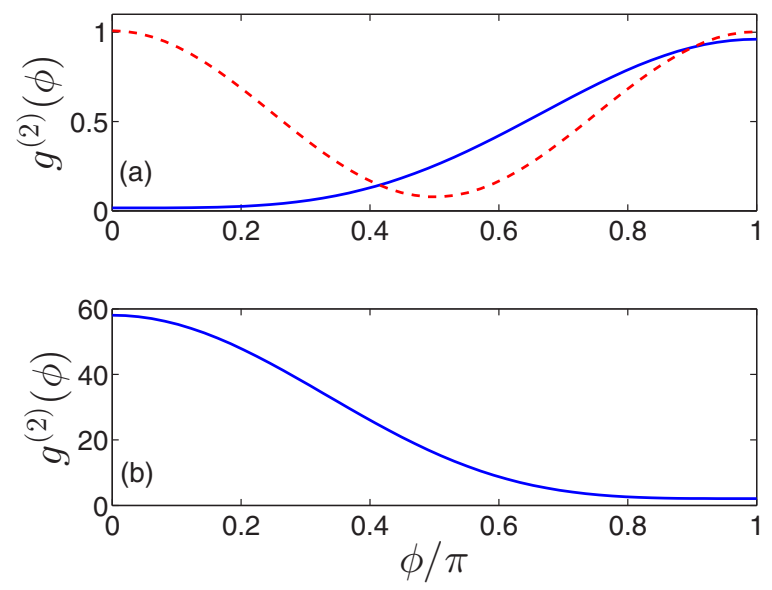

FIG. 5. (Color online) Equal-time second-order correlation function $g^{(2)}\left(r_{\circ}=0.5 \ell, \phi\right)$ in the steady state for different driving frequencies of the $\mathrm{LG}_{0}^{1}$ pump. (a) $\Delta \omega_{p} / \omega=2$ (solid blue line), $\Delta \omega_{p} / \omega \approx 2.004$ (dashed red line). (b) $\Delta \omega_{p} / \omega \approx 2.008$. System and pump parameters: $g_{\mathrm{nl}} / \ell^{2} \omega=0.1, \gamma / \omega=0.002$, and $\ell F / \gamma=0.1$. 


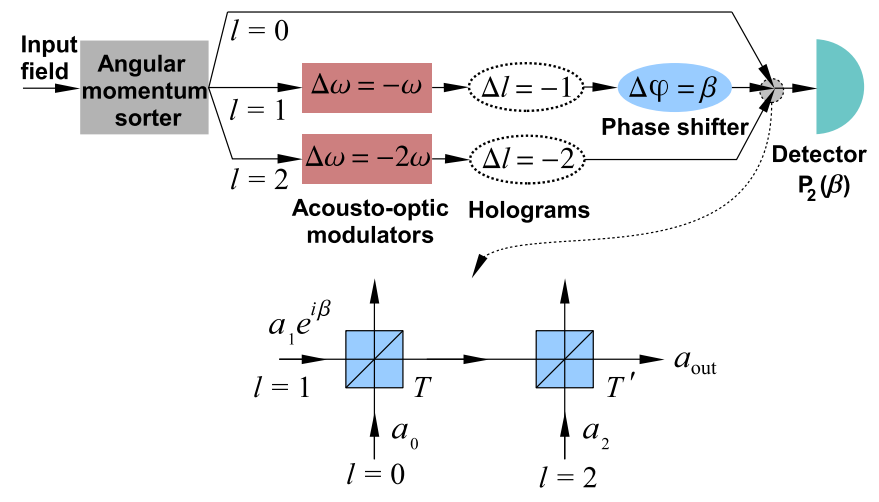

FIG. 6. (Color online) Sketch of the experiment proposed to distinguish between the two-particle Laughlin and c.m. states by measuring the two-particle detection probability $P_{2}$ as a function of $\beta$.

$r_{\circ} \sim \ell$ (cf. Appendix D). Although $\left|c_{2} / c_{1}\right|$ remains essentially the same for two different driving frequencies, $\left|c_{1}\right|$ is reduced by a factor of $\sim 8$ for $\Delta \omega_{p} / \omega \approx 2.008$ as it is displaced from the single-particle resonance at $\Delta \omega_{p} / \omega=2$. Also shown in Fig. 5(a) by dashed lines is $g^{(2)}(\phi)$ for $\omega_{p} / \omega \approx 2.004$ when an equal-weight superposition of Laughlin and c.m. states is nonresonantly excited [see Fig. 2(b)]. In this case, $g^{(2)}(\phi)$ also shows a hybrid behavior. In all three cases, Eq. (13) fits perfectly to the numerical data of Fig. 5. Similar results were obtained for the resonant excitation of the three-particle Laughlin and c.m. states using an $\mathrm{LG}_{0}^{2}$ drive (cf. Appendix E).

Before concluding the section, it is interesting to mention also another scheme which may be used to differentiate the two-particle Laughlin state $\left|\psi_{-}\right\rangle=(|101\rangle-|020\rangle) / \sqrt{2}$ from the c.m. state $\left|\psi_{+}\right\rangle=(|101\rangle+|020\rangle) / \sqrt{2}$ using photodetectors without spatial resolution. The principle of the method is sketched in Fig. 6: After the AOM stage, instead of recombining all beams immediately, one may use $\Delta l=$ $-1,-2$ holograms to bring all components (including the initially $l=1,2$ ones) to the $l=0$ state, in order for the beams to efficiently interfere. Two symmetric beam splitters with possibly different transmissivities $T, T^{\prime}$ are then used to mix the three beams and the joint probability of detecting two photons on the same output arm is finally measured in a kind of Hong-Ou-Mandel two-photon interference scheme [34]. Describing the output mode as a superposition of the annihilation operators for the initial states as $a_{\text {out }}=\left(i \sqrt{1-T} a_{0}+\right.$ $\left.e^{i \beta} \sqrt{T} a_{1}\right) \sqrt{T^{\prime}}+i \sqrt{1-T^{\prime}} a_{2}$, this probability is found to be $P_{2}(\beta) \propto\left\langle\psi_{\mp}\left|a_{\text {out }}^{\dagger} a_{\text {out }}^{\dagger} a_{\text {out }} a_{\text {out }}\right| \psi_{\mp}\right\rangle \propto(5 \pm 4 \cos 2 \beta)$ for $T=$ $T^{\prime}=1 / 2$, which depends on the (adjustable) phase shift $\beta$ imposed to the initial $l=1$ beam and allows one to differentiate the two strongly correlated states of light.

\section{CONCLUSION}

We have shown that strongly correlated Laughlin states of interacting photons can be prepared by shining a weak Laguerre-Gauss beam of light onto a single optically nonlinear cavity enclosed by curved mirrors, which supports a hierarchy of transverse modes analogous to the eigenstates of a twodimensional harmonic oscillator. By focusing on a definite particle number $N$ via a coincidence rate measurement, different strongly correlated states are seen to appear as resonances in the transmission spectrum of the device. In addition to the full reconstruction of the $N$-photon amplitude via homodyne techniques as proposed in our previous work, here we show how simpler measurements of the power emission spectrum and of the second-order correlation functions already provide evidence for the existence of strong correlations, with features substantially different from those of weakly interacting particles.

\section{ACKNOWLEDGMENTS}

R.O.U. is supported by the FWO through a Pegasus Marie Curie Fellowship. M.W. and R.O.U. acknowledge financial support from the FWO through the Odysseus Programme. I.C. acknowledges partial financial support from ERC via the QGBE grant and from the Autonomous Province of Trento, Call "Grandi Progetti 2012," project “On silicon chip quantum optics for quantum computing and secure communications SiQuro." Support from the POLATOM ESF network is also acknowledged. We are grateful to T. Volz, G. Molina-Terriza, and J. Simon for useful discussions.

\section{APPENDIX A: ENERGY CONSIDERATIONS FOR THE LAUGHLIN AND CENTER-OF-MASS STATES}

We numerically verified for $N=2,3$, and 4 that the highest-energy eigenfunction in the lowest Landau band with total angular momentum $L_{z}=N(N-1) \hbar$ is proportional to $\left(z_{1}+\cdots+z_{N}\right)^{N(N-1)}$ corresponding to pure center-of-mass (c.m.) rotation. This result can be understood intuitively as follows. The many-body wave function for a system in a harmonic potential with two-body interactions can be written as a product of two wave functions, one depending on the c.m. coordinate $\left(z_{1}+\cdots+z_{N}\right)$ and one on relative coordinates $\left(z_{i}-z_{j}\right.$ ) (see, e.g., Ref. [35]), a fact which leads to a generalized form [36,37] of the Kohn's theorem [38]. Since the kinetic energy is fixed in the LLL, the highest-energy eigenfunction will be the one with the largest interaction energy. The effect of the presence of relative coordinates in the eigenfunction is to reduce the interaction energy as the contribution of any term containing a relative coordinate vanishes for a contact interaction $\propto \sum_{i<j} \delta\left(z_{i}-z_{j}\right)$. Therefore the interaction energy is maximized if the relative part of the many-body wave function is simply a constant and as a result all the angular momentum is transferred to the center of mass.

We now wish to find the width of the lowest Landau band defined as the difference between the energies of the Laughlin and c.m. states. We will start with the simplest two-particle case. As the kinetic energy is fixed we will only consider the interaction Hamiltonian:

$$
\mathcal{H}_{\text {int }}=\frac{1}{2} \iint \hat{\Psi}^{\dagger}(\mathbf{r}) \hat{\Psi}^{\dagger}\left(\mathbf{r}^{\prime}\right) V\left(\mathbf{r}-\mathbf{r}^{\prime}\right) \hat{\Psi}\left(\mathbf{r}^{\prime}\right) \hat{\Psi}(\mathbf{r}) d^{2} \mathbf{r} d^{2} \mathbf{r}^{\prime}
$$

For a contact interaction $V\left(\mathbf{r}-\mathbf{r}^{\prime}\right)=\hbar g_{\mathrm{nl}} \delta^{(2)}\left(\mathbf{r}-\mathbf{r}^{\prime}\right)$, expanding $\hat{\Psi}(\mathbf{r})=\sum_{m} \varphi_{m}(\mathbf{r}) a_{m}$ in the LLL basis functions $\varphi_{m}(\mathbf{r})=$ $|\mathbf{r}|^{m} e^{i m \theta} e^{-|\mathbf{r}|^{2} / 2 \ell^{2}} / \sqrt{\pi m ! \ell^{2(m+1)}}$, with $\ell=\sqrt{\hbar / m_{\mathrm{ph}} \omega}$, we find 
the interaction Hamiltonian to be

$$
\mathcal{H}_{\text {int }}=\frac{1}{2} \sum_{i j k l} V_{i j k l} a_{i}^{\dagger} a_{j}^{\dagger} a_{k} a_{l}
$$

with $V_{i j k l}=\hbar g_{\mathrm{nl}} \int \varphi_{i}^{*}(\mathbf{r}) \varphi_{j}^{*}(\mathbf{r}) \varphi_{k}(\mathbf{r}) \varphi_{l}(\mathbf{r}) d^{2} \mathbf{r}$. Using $\left|\psi_{\mp}\right\rangle=$ $(|101\rangle \mp|020\rangle) / \sqrt{2}$ for the two-particle Laughlin (-) and c.m. (+) states, the interaction energy is calculated as

$$
\begin{aligned}
& \left\langle\psi_{\mp}\left|\mathcal{H}_{\text {int }}\right| \psi_{\mp}\right\rangle \\
& \quad=\frac{1}{4}\left(4 V_{0202} \mp 2 \sqrt{2} V_{0211} \mp 2 \sqrt{2} V_{1102}+2 V_{1111}\right) .
\end{aligned}
$$

Thus the energy gap between these two states is

$$
\begin{aligned}
\Delta & =\left\langle\psi_{+}\left|\mathcal{H}_{\text {int }}\right| \psi_{+}\right\rangle-\left\langle\psi_{-}\left|\mathcal{H}_{\text {int }}\right| \psi_{-}\right\rangle \\
& =\sqrt{2}\left(V_{0211}+V_{1102}\right)=2 \sqrt{2} V_{0211}=\frac{\hbar g_{\mathrm{nl}}}{2 \pi \ell^{2}} .
\end{aligned}
$$

The generalization of this result to the $N$-particle case is most easily done in first quantization by calculating

$$
\begin{aligned}
\left\langle\mathcal{H}_{\text {int }}\right\rangle_{\text {c.m. }}= & \frac{\hbar g_{\mathrm{nl}}}{\ell^{2}} \int \sum_{i<j} \delta\left(z_{i}-z_{j}\right)\left|\Psi_{\text {c.m. }}\left(z_{1}, \ldots, z_{N}\right)\right|^{2} \\
& \times d z_{1} d z_{1}^{*} \cdots d z_{N} d z_{N}^{*}
\end{aligned}
$$

for the many-body wave function $\Psi_{\text {c.m. }}\left(z_{1}, \ldots, z_{N}\right)=\mathcal{A}\left(z_{1}+\right.$ $\left.\cdots+z_{N}\right)^{L} e^{-\sum_{n=1}^{N}\left|z_{n}\right|^{2} / 2}$, where $z_{n}=\left(x_{n}+i y_{n}\right) / \ell$ is the complex coordinate of the $n$th particle, $L=N(N-1)$, and $\mathcal{A}$ is a normalization constant. Integrating over the coordinate of a particle in the argument of the $\delta$ function and summing over all distinct pairs, Eq. (A5) becomes

$$
\begin{aligned}
\left\langle\mathcal{H}_{\text {int }}\right\rangle_{\text {c.m. }}= & \frac{\hbar g_{\text {nl }}}{\ell^{2}} \frac{N(N-1)}{2}|\mathcal{A}|^{2} \int\left(2 z_{2}^{*}+\cdots+z_{N}^{*}\right)^{L} \\
& \times\left(2 z_{2}+\cdots+z_{N}\right)^{L} d\{z\} d\left\{z^{*}\right\},
\end{aligned}
$$

where $d\{z\} \equiv e^{-\left|z_{2}\right|^{2}-\sum_{n=3}^{N}\left|z_{n}\right|^{2} / 2} d z_{2} \cdots d z_{N}$. Now we use the multinomial theorem:

$$
\left(X_{1}+\cdots+X_{m}\right)^{n}=\sum_{k_{1}+\cdots+k_{m}=n} \frac{n !}{k_{1} ! \cdots k_{m} !} X_{1}^{k_{1}} \cdots X_{m}^{k_{m}}
$$

and write Eq. (A6) as

$$
\begin{aligned}
\left\langle\mathcal{H}_{\text {int }}\right\rangle_{\text {c.m. }}= & \frac{\hbar g_{\mathrm{nl}}}{\ell^{2}} \frac{N(N-1)}{2}|\mathcal{A}|^{2} \\
& \times \sum_{\substack{k_{2}+\cdots+k_{N}=L \\
k_{2}^{\prime}+\cdots+k_{N}^{\prime}=L}} \frac{L !}{k_{2} ! \cdots k_{N} !} \frac{L !}{k_{2}^{\prime} ! \cdots k_{N}^{\prime} !} \\
& \times \int\left(2 z_{2}^{*}\right)^{k_{2}}\left(2 z_{2}\right)^{k_{2}^{\prime}} \cdots\left(z_{N}^{*}\right)^{k_{N}}\left(z_{N}\right)^{k_{N}^{\prime}} d\{z\} d\left\{z^{*}\right\} .
\end{aligned}
$$

For the integral over the angular part of a coordinate $z_{n}$ not to vanish, we must have $k_{n}=k_{n}^{\prime}$. Using this fact and performing the integrations $\int\left|z_{n}\right|^{2 k_{n}} e^{-\left|z_{n}\right|^{2}} d z_{n} d z_{n}^{*}=\pi k_{n}$ !, $\int\left|2 z_{2}\right|^{2 k_{2}} e^{-2\left|z_{2}\right|^{2}} d z_{2} d z_{2}^{*}=2^{k_{2}} k_{2} ! \pi / 2$, Eq. (A8) becomes

$$
\begin{aligned}
\left\langle\mathcal{H}_{\text {int }}\right\rangle_{\text {c.m. }}= & \frac{\hbar g_{\mathrm{nl}}}{\ell^{2}} \frac{N(N-1)}{2}|\mathcal{A}|^{2} \frac{\pi^{N-1}}{2} L ! \\
& \times \sum_{k_{2}+\cdots+k_{N}=L} \frac{L !}{k_{2} ! \cdots k_{N} !} 2^{k_{2}} 1^{k_{3}} \cdots 1^{k_{N}} .
\end{aligned}
$$

The summation in Eq. (A9) can be evaluated by using Eq. (A7) to yield $N^{L}$. Through similar steps the normalization constant can be found to be $\mathcal{A}=1 / \sqrt{\pi^{N} L ! N^{L}}$. Inserting these results into Eq. (A9), the interaction energy of the $N$-particle c.m. state is finally given by

$$
\left\langle\mathcal{H}_{\text {int }}\right\rangle_{\text {c.m. }}=\frac{\hbar g_{\mathrm{nl}}}{\ell^{2}} \frac{N(N-1)}{2} \frac{1}{2 \pi}=\frac{N(N-1)}{2} \Delta,
$$

which is also the width of the lowest Landau band since the interaction energy of the Laughlin state is zero. This result has also been numerically verified for $N=2,3$, and 4 . The energy gap between the Laughlin state and the first excited state with the same total angular momentum is a fraction of the bandwidth and close to $\Delta$. The two-particle gap $\Delta$ actually corresponds to the lowest Haldane pseudopotential $v_{0}$ for the contact interaction, as the $m$ th Haldane pseudopotential $v_{m}$ for the LLL is defined to be the expected value of the interaction energy with respect to the normalized wave function $\mathcal{N}\left(z_{1}-z_{2}\right)^{m}\left(z_{1}+z_{2}\right)^{M} e^{-\left(\left|z_{1}\right|^{2}+\left|z_{2}\right|^{2}\right) / 2}$, where two particles have relative angular momentum $m \hbar[27,31]$. Note that the value of the pseudopotential does not depend on the center-of-mass momentum $M \hbar$.

\section{APPENDIX B: LOSS OF A PARTICLE FROM THE LAUGHLIN AND CENTER-OF-MASS STATES}

The resultant state after a particle with angular momentum $l \hbar$ is annihilated from a general bosonic $N$-particle state $\left|\Phi_{N}\right\rangle$ lying in the LLL can be found by applying $a_{l}=\int d z^{\prime} d z^{* *} \varphi_{l}^{*}\left(z^{\prime}\right) \hat{\Psi}\left(z^{\prime}\right)$ to $\left|\Phi_{N}\right\rangle=$ $\int d z_{1} d z_{1}^{*} \cdots d z_{N} d z_{N}^{*} \Phi_{N}\left(z_{1}, \ldots, z_{N}\right) \hat{\Psi}^{\dagger}\left(z_{1}\right) \cdots \hat{\Psi}^{\dagger}\left(z_{N}\right)|\mathrm{vac}\rangle$ $|\mathrm{vac}\rangle$ being the vacuum state, which yields

$$
\begin{aligned}
\left|\Phi_{N-1}^{\prime}\right\rangle \equiv & a_{l}\left|\Phi_{N}\right\rangle \\
= & N \int d z_{1} d z_{1}^{*} \cdots d z_{N-1} d z_{N-1}^{*} \\
& \times\left[\int \Phi_{N}\left(z_{1}, \ldots, z_{N-1}, z\right) \varphi_{l}^{*}(z) d z d z^{*}\right] \\
& \times \hat{\Psi}^{\dagger}\left(z_{1}\right) \ldots \hat{\Psi}^{\dagger}\left(z_{N-1}\right)|\mathrm{vac}\rangle
\end{aligned}
$$

The wave function corresponding to the resultant state with $N-1$ particles is identified as $\Phi_{N-1}^{\prime}\left(z_{1}, \ldots, z_{N-1}\right)=$ $\int \Phi_{N}\left(z_{1}, \ldots, z_{N-1}, z\right) \varphi_{l}^{*}(z) d z d z^{*}$ up to a normalization constant. Choosing, for instance, $z=z_{N}$ and noting that $\varphi_{l}^{*}\left(z_{N}\right) \propto$ $z_{N}^{* l}, \Phi_{N-1}^{\prime}$ is found to be proportional to the multinomial term multiplying $z_{N}^{l}$ in $\Phi_{N}$ as it is the only surviving term in the integral expression for $\Phi_{N-1}^{\prime}$.

For the Laughlin wave function (4) it is easy to see that $\Phi_{N-1}^{\prime}$ is the $(N-1)$-particle Laughlin wave function times a symmetric polynomial in coordinates $\left\{z_{1}, \ldots, z_{N-1}\right\}$ with total power $2(N-1)-l$. For the c.m. wave function (5), using the 
multinomial expansion (A7), it can be found that $\Phi_{N-1}^{\prime} \propto$ $\left(z_{1}+\cdots+z_{N-1}\right)^{N(N-1)-l}$.

\section{APPENDIX C: POWER SPECTRUM FOR THE RESONANT EXCITATION OF THE CENTER-OF-MASS STATE}

The simulated power spectrum for the resonant excitation of the two-particle c.m. state is shown in Fig. 7. In addition to the pump peak at $\omega_{s} / \omega \approx 2.008$, there are two more peaks visible at $\omega_{s} / \omega=1,1+\Delta / \hbar \omega \approx 1.016$, where $\Delta / \hbar \omega \approx 0.016$ is fixed by Eq. (A4). While the peak at $\omega_{s} / \omega=1+\Delta / \hbar \omega$ is due to the transition from the two-particle c.m. state to the single-particle state with angular momentum $2 \hbar$ without change in total angular momentum, the peak with almost equal amplitude at $\omega_{s} / \omega=1$ is caused by the transition from the single-particle state with zero angular momentum (which is present because of the quantum jump from the two-particle c.m. state) to the vacuum.

\section{APPENDIX D: SECOND-ORDER CORRELATION FUNCTION FOR THE RESONANT EXCITATION OF TWO-PARTICLE STATES}

The full result for the normalized equal-time second-order correlation function calculated using Eqs. (6) and (7) of the main text is

$$
\begin{aligned}
g^{(2)}\left(r_{\circ}, \phi\right)= & \alpha\left(r_{\circ}\right)\left[(1+\cos 2 \phi)|a+b|^{2}\right. \\
& \left.+4 \cos \phi\left(b^{2}-|a|^{2}\right)+2|a-b|^{2}\right],
\end{aligned}
$$

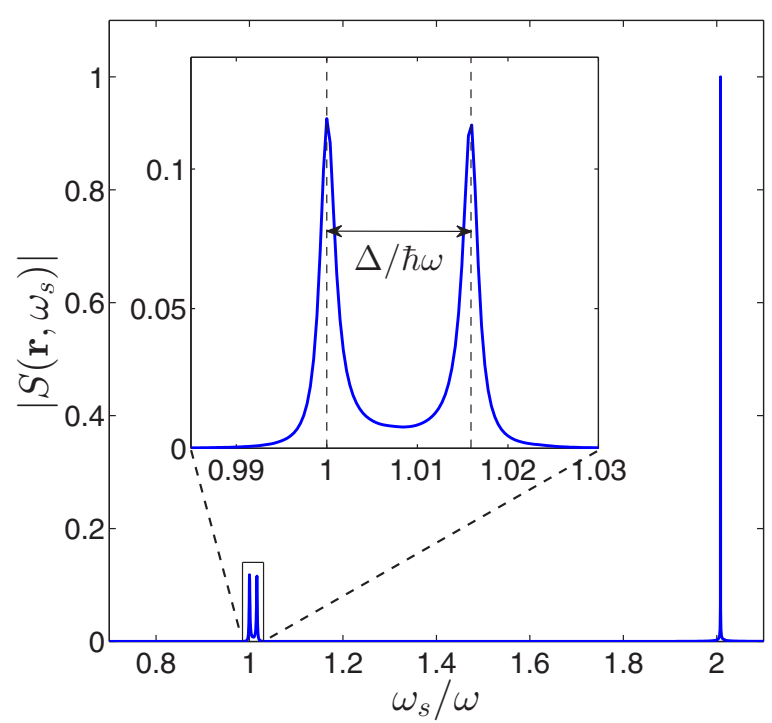

FIG. 7. (Color online) Absolute value of the power spectrum $\left|S\left(\mathbf{r}, \omega_{s}\right)\right|$ in arbitrary units as a function of frequency $\omega_{s}$ measured with respect to the cavity frequency $\omega_{c}$; taking the absolute value smooths out the effect of the finite time window used in the numerical calculation on the $\delta$-shaped pump peak. The inset is a closeup around $\omega_{s} / \omega=1$ showing the double-peak structure. The system is pumped by a Laguerre-Gauss $\mathrm{LG}_{0}^{1}$ beam at $\Delta \omega_{p} / \omega \approx 2.008 ;|\mathbf{r}|=0.05 \ell$. System and pump parameters: $g_{n l} / \ell^{2} \omega=0.1, \gamma / \omega=0.002$, and $\ell F / \gamma=0.1$.

with

$$
\alpha\left(r_{\circ}\right)=\frac{\left|c_{2} \tilde{F}^{2}\right|^{2} \tilde{r}_{\circ}^{4}}{\left\{\left(1+\frac{\tilde{r}_{0}^{4}}{2}\right)(1+\mathcal{N})\left|c_{2} \tilde{F}^{2}(a+b)\right|^{2}+\tilde{r}_{\circ}^{2}\left[\left|c_{1} \tilde{F}\right|^{2}+2\left|c_{2} \tilde{F}^{2}(a-b)\right|^{2}(1+\mathcal{N})\right]\right\}^{2}},
$$

where $\tilde{r}_{\circ} \equiv r_{\circ} / \ell$. For $\tilde{r}_{\circ} \sim 1$, the denominator is approximately $\left|c_{1} \tilde{F}\right|^{4} \tilde{r}_{\circ}^{4}$ since $\tilde{F} \ll 1$. Hence $\alpha\left(\tilde{r}_{\circ} \sim 1\right) \sim\left|c_{2}\right|^{2} /\left|c_{1}\right|^{4}$.

For comparison with Fig. 5, we also show in Fig. 8 $g^{(2)}(\phi) \propto 3+\cos 2 \phi \mp 4 \cos \phi$ calculated for the pure twoparticle Laughlin $(-)$ and c.m. $(+)$ states $(|101\rangle \mp|020\rangle) / \sqrt{2}$.

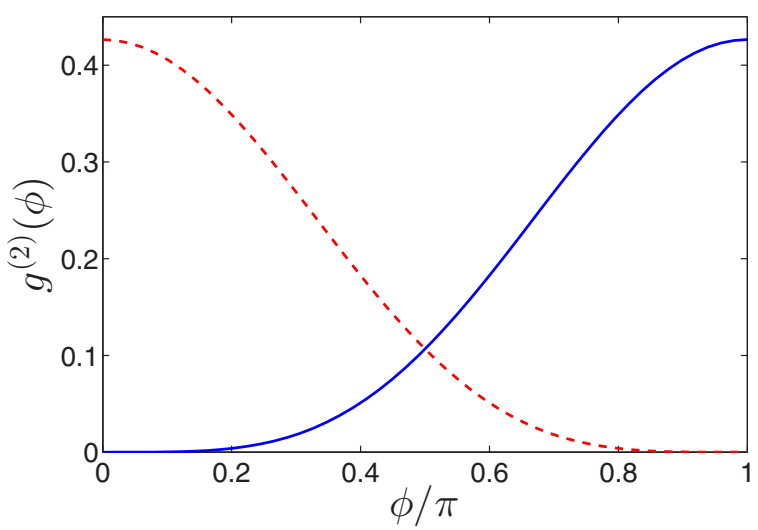

FIG. 8. (Color online) Solid (dashed) line shows $g^{(2)}\left(r_{\circ}=\right.$ $0.5 \ell, \phi)$ for the normalized two-particle Laughlin (c.m.) wave function.

\section{APPENDIX E: SECOND-ORDER CORRELATION FUNCTION FOR THE RESONANT EXCITATION OF THREE-PARTICLE STATES}

In order to show that the marked difference between the Laughlin and c.m. states persists also in the resonant excitation of three-particle eigenstates, we display in Fig. 9 the second-order correlation function $g^{(2)}(\phi)$ numerically calculated for seven different driving frequencies of the $\mathrm{LG}_{0}^{2}$ pump, corresponding to the lowest lying eigenfrequencies of the three-particle eigenstates with total angular momentum $6 \hbar$ of the isolated system. Note that the number of eigenstates is set by the seven distinct ways to distribute a total angular momentum of $6 \hbar$ to three particles in the LLL. For completeness we give the explicit expressions for the unnormalized eigenfunctions (without displaying the ubiquitous exponential factor) found by numerical diagonalization:

$$
\text { |1) } \propto z_{12}^{2} z_{13}^{2} z_{23}^{2}
$$

$$
|2\rangle \propto\left(z_{12}+z_{13}\right)\left(z_{13}+z_{23}\right)\left(z_{12}-z_{23}\right) Z^{3},
$$

$|3\rangle \propto\left(z_{12}+z_{13}\right)\left(z_{13}+z_{23}\right)\left(z_{12}-z_{23}\right)\left(z_{12}^{2}+z_{13}^{2}+z_{23}^{2}\right) Z$, 


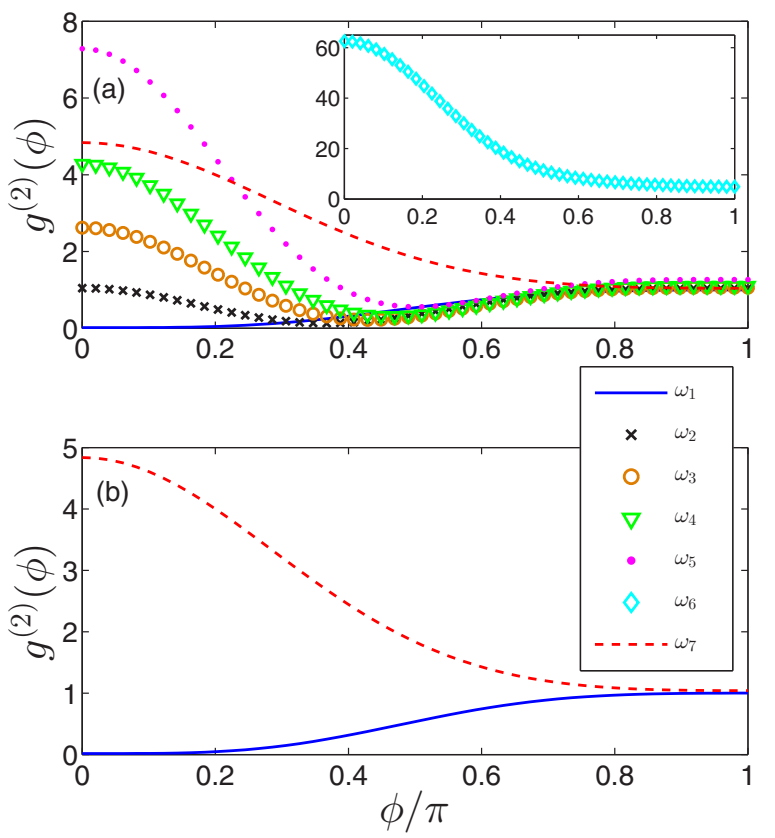

FIG. 9. (Color online) (a) $g^{(2)}\left(r_{\circ}=0.5 \ell, \phi\right)$ for seven different frequencies of the $\mathrm{LG}_{0}^{2}$ pump corresponding to the eigenfrequencies of the isolated system. Frequency index increases with increasing energy eigenvalue. (b) $g^{(2)}\left(r_{\circ}=0.5 \ell, \phi\right)$ for $\Delta \omega_{p} / \omega=3$ (solid blue line) corresponding to the Laughlin state and $\Delta \omega_{p} / \omega \approx 3.00159$ (dashed red line) corresponding to the c.m. state, plotted again for clarity. System and pump parameters: $g_{n l} / \ell^{2} \omega=0.1, \gamma / \omega=0.002$, and $\ell F / \gamma=0.1$.

$$
\begin{aligned}
&|4\rangle \propto 7\left(z_{12}^{6}+z_{13}^{6}+z_{23}^{6}\right) \\
&- 15\left(z_{12}^{4}+z_{13}^{4}+z_{23}^{4}\right)\left(z_{12}^{2}+z_{13}^{2}+z_{23}^{2}\right) \\
&- 20\left(z_{12}^{3} z_{13}^{3}+z_{13}^{3} z_{23}^{3}-z_{12}^{3} z_{23}^{3}\right), \\
&|5\rangle \propto\left(z_{12}^{2}+z_{13}^{2}+z_{23}^{2}\right)^{2} Z^{2}, \\
&|6\rangle \propto\left(z_{12}^{2}+z_{13}^{2}+z_{23}^{2}\right) Z^{4},
\end{aligned}
$$$$
|7\rangle \propto Z^{6},
$$

where $z_{12}=z_{1}-z_{2}, z_{13}=z_{1}-z_{3}, z_{23}=z_{2}-z_{3}$ are the relative coordinates and $Z=\left(z_{1}+z_{2}+z_{3}\right) / 3$ is the c.m. coordinate. The trend we observed in the resonant excitation of two-particle Laughlin and c.m. states is also seen in this case as clearly shown in Fig. 9(b). Because of the weak-driving condition $g^{(2)}$ will be dominated by the two-particle sector of the steady state, in particular by those terms that do not involve additional quantum jump terms. When targeting the three-particle Laughlin state, the two-particle wave function is numerically found to be a linear superposition of wave functions $\left(z_{1}-z_{2}\right)^{2}\left(z_{1}^{2}+z_{2}^{2}\right)$ and $\left(z_{1}-z_{2}\right)^{2} z_{1} z_{2}$. As a result, $g^{(2)}$ still reflects the behavior obtained for the two-particle Laughlin state.

On the other hand, when the three-particle c.m. state is excited, the two-particle wave function turns out to be a superposition of wave functions $\left(z_{1}+z_{2}\right)^{4},\left(z_{1}-z_{2}\right)^{2}\left(z_{1}^{2}+\right.$ $\left.z_{2}^{2}\right)$, and $\left(z_{1}-z_{2}\right)^{2} z_{1} z_{2}$, its overlap with the two-particle c.m. wave function $\left(z_{1}+z_{2}\right)^{4}$ being $70 \%$, which results in a $g^{(2)}$ profile similar to the one obtained for the direct excitation of the two-particle c.m. state with a total angular momentum of $2 \hbar$.
[1] N. R. Cooper, Adv. Phys. 57, 539 (2008).

[2] A. L. Fetter, Rev. Mod. Phys. 81, 647 (2009).

[3] J. Dalibard, F. Gerbier, G. Juzeliūnas, and P. Öhberg, Rev. Mod. Phys. 83, 1523 (2011).

[4] N. Goldman, G. Juzeliūnas, P. Öhberg, and I. B. Spielman, arXiv:1308.6533.

[5] I. Carusotto and C. Ciuti, Rev. Mod. Phys. 85, 299 (2013).

[6] F. D. M. Haldane and S. Raghu, Phys. Rev. Lett. 100, 013904 (2008).

[7] Z. Wang et al., Nature (London) 461, 772 (2009).

[8] J. Cho, D. G. Angelakis, and S. Bose, Phys. Rev. Lett. 101, 246809 (2008).

[9] A. L. C. Hayward, A. M. Martin, and A. D. Greentree, Phys. Rev. Lett. 108, 223602 (2012).

[10] J. Koch, A. A. Houck, K. L. Hur, and S. M. Girvin, Phys. Rev. A 82, 043811 (2010).

[11] M. Hafezi et al., Nat. Phys. 7, 907 (2011).

[12] M. Hafezi et al., Nat. Photon. 7, 1001 (2013).

[13] J. Keeling, Phys. Rev. Lett. 107, 080402 (2011).

[14] R. O. Umucalılar and I. Carusotto, Phys. Rev. A 84, 043804 (2011).

[15] K. Fang, Z. Yu, and S. Fan, Nat. Photon. 6, 782 (2012).

[16] M. C. Rechtsman et al., Nat. Photon. 7, 153 (2013).

[17] M. C. Rechtsman et al., Nature (London) 496, 196 (2013).

[18] A. Nunnenkamp et al., New. J. Phys. 13, 095008 (2011).
[19] R. O. Umucalılar and I. Carusotto, Phys. Rev. Lett. 108, 206809 (2012).

[20] M. Hafezi, M. D. Lukin, and J. M. Taylor, New J. Phys. 15, 063001 (2013).

[21] T. Peyronel et al., Nature (London) 488, 57 (2012).

[22] P. Cristofolini et al., Science 336, 704 (2012).

[23] R. O. Umucalılar and I. Carusotto, Phys. Lett. A 377, 2074 (2013).

[24] N. K. Wilkin, J. M. F. Gunn, and R. A. Smith, Phys. Rev. Lett. 80, 2265 (1998).

[25] B. Paredes, P. Fedichev, J. I. Cirac, and P. Zoller, Phys. Rev. Lett. 87, 010402 (2001).

[26] R. B. Laughlin, Phys. Rev. Lett. 50, 1395 (1983).

[27] D. Yoshioka, The Quantum Hall Effect (Springer, Berlin, 2002).

[28] J. Klaers, J. Schmitt, F. Vewinger, and M. Weitz, Nature (London) 468, 545 (2010).

[29] C. W. Gardiner and P. Zoller, Quantum Noise, 2nd ed. (Springer, Berlin, 2000).

[30] L. D. Landau and E. M. Lifshitz, Mechanics, 3rd ed. (Butterworth-Heinemann, Oxford, 2000).

[31] S. M. Girvin, The Quantum Hall Effect: Novel Excitations and Broken Symmetries, in Topological Aspects of Low Dimensional Systems, edited by A. Comtet, T. Jolicoeur, S. Ouvry, and F. David (Springer, Berlin and Les Editions de Physique, Les Ulis, 2000). 
[32] M. O. Scully and M. S. Zubairy, Quantum Optics (Cambridge University Press, Cambridge, 1997).

[33] J. Leach, J. Courtial, K. Skeldon, S. M. Barnett, S. Franke-Arnold, and M. J. Padgett, Phys. Rev. Lett. 92, 013601 (2004); H. Wei et al., Opt. Commun. 223, 117 (2003); J. Leach, M. J. Padgett, S. M. Barnett, S. FrankeArnold, and J. Courtial, Phys. Rev. Lett. 88, 257901 (2002).
[34] C. K. Hong, Z. Y. Ou, and L. Mandel, Phys. Rev. Lett. 59, 2044 (1987).

[35] P. Bakshi, D. A. Broido, and K. Kempa, Phys. Rev. B 42, 7416 (1990).

[36] L. Brey, N. F. Johnson, and B. I. Halperin, Phys. Rev. B 40, 10647 (1989).

[37] S. K. Yip, Phys. Rev. B 43, 1707 (1991).

[38] W. Kohn, Phys. Rev. 123, 1242 (1961). 